\title{
Impact of ${ }^{18}$ F-PSMA-1007 Uptake in Prostate Cancer Using Different Peptide Concentrations: Preclinical PET/CT Study on Mice
}

\author{
Fumihiko Soeda ${ }^{1}$, Tadashi Watabe ${ }^{1}$, Sadahiro $\mathrm{Naka}^{2}$, Yuwei Liu ${ }^{1}$, Genki Horitsugi ${ }^{1}$, Oliver C. Neels ${ }^{3}$, Klaus Kopka ${ }^{3,4}$, \\ Mitsuaki Tatsumi ${ }^{5}$, Eku Shimosegawa ${ }^{6}$, Frederik L. Giesel ${ }^{7,4}$, and Jun Hatazawa ${ }^{1}$ \\ ${ }^{I}$ Department of Nuclear Medicine and Tracer Kinetics, Osaka University Graduate School of Medicine, Osaka, Japan; ${ }^{2}$ Department \\ of Pharmaceuticals, Osaka University Hospital, Osaka, Japan; ${ }^{3}$ Division of Radiopharmaceutical Chemistry, German Cancer \\ Research Center, Heidelberg, Germany; ${ }^{4}$ German Cancer Consortium, Heidelberg, Germany; ${ }^{5}$ Department of Radiology, Osaka \\ University Hospital, Osaka, Japan; ${ }^{6}$ Department of Molecular Imaging in Medicine, Osaka University Graduate School of Medicine, \\ Osaka, Japan; and ${ }^{7}$ Department of Nuclear Medicine, University Hospital Heidelberg, Heidelberg, Germany
}

PET radioligands with low molar activity (MA) may underestimate the quantity of the target of interest because of competitive binding of the target with unlabeled ligand. The aim of this study was to evaluate the change in the whole-body distribution of ${ }^{18} \mathrm{~F}-\mathrm{PSMA}$ 1007 targeting prostate-specific membrane antigen (PSMA) when solutions with different peptide concentrations are used. Methods: Mouse xenograft models of LNCaP (PSMA-positive prostate cancer) $(n=18)$ were prepared and divided into 3 groups according to the peptide concentration injected: a high-MA group $(1,013 \pm 146$ $\mathrm{GBq} / \mu \mathrm{mol} ; n=6)$, a medium-MA group $(100.7 \pm 23.1 \mathrm{GBq} / \mu \mathrm{mol} ;$ $n=6)$, and a low-MA group (10.80 $\pm 2.84 \mathrm{GBq} / \mu \mathrm{mol} ; n=6)$. Static PET scans were performed $1 \mathrm{~h}$ after injection (scan duration, $10 \mathrm{~min}$ ). SUV $\mathrm{V}_{\text {mean }}$ in tumor and normal organs was compared by the multiple-comparison test. Immunohistochemical staining and Western blot analysis were performed to confirm expression of PSMA in tumor, salivary gland, and kidney. Results: The low-MA group $\left(S_{U} V_{\text {mean }}, 1.12 \pm 0.30\right)$ showed significantly lower uptake of ${ }^{18} \mathrm{~F}-\mathrm{PSMA}-1007$ in tumor than did the high-MA group $(1.97 \pm 0.77)$ and the medium-MA group $(1.81 \pm 0.57)$. On the other hand, in salivary gland, both the low-MA group $\left(\mathrm{SUV}_{\text {mean }}, 0.24 \pm 0.04\right)$ and the medium-MA group $(0.57 \pm 0.08)$ showed significantly lower uptake than the high MA group (1.27 \pm 0.28$)$. The tumor-to-salivary gland SUV $_{\text {mean }}$ ratio was $1.73 \pm 0.55$ in the high-MA group, $3.16 \pm$ 0.86 in the medium-MA group, and $4.78 \pm 1.29$ in the low-MA group. The immunohistochemical staining and Western blot analysis revealed significant overexpression of PSMA in tumor and low expression in salivary gland and kidney. Conclusion: A decrease in the MA level of the injected ${ }^{18} \mathrm{~F}-\mathrm{PSMA}-1007$ solution resulted in decreased uptake in tumor and, to a greater degree, in normal salivary gland. Thus, there is a possibility of minimizing the adverse effects in salivary gland by setting an appropriate MA level in PSMAtargeting therapy.

Received Nov. 21, 2018; revision accepted Mar. 13, 2019.

For correspondence or reprints contact: Tadashi Watabe, Osaka University Graduate School of Medicine, 2-2 Yamadaoka, Suita, Osaka 565-0871 Japan.

E-mail: watabe@tracer.med.osaka-u.ac.jp

Published online Mar. 22, 2019.

Immediate Open Access: Creative Commons Attribution 4.0 International License (CC BY) allows users to share and adapt with attribution, excluding materials credited to previous publications. License: https://creativecommons. org/licenses/by/4.0/. Details: http://jnm.snmjournals.org/site/misc/permission. xhtml.

COPYRIGHT @ 2019 by the Society of Nuclear Medicine and Molecular Imaging.
Key Words: ${ }^{18} \mathrm{~F}-\mathrm{PSMA}-1007$; peptide concentration; molar activity; prostate cancer; salivary gland

J Nucl Med 2019; 60:1594-1599

DOI: 10.2967/jnumed.118.223479

$\mathbf{P}$ rostate-specific membrane antigen (PSMA) is overexpressed in prostate cancer cells and is considered a novel target of theranostics in prostate cancer $(1,2)$. It is used as an effective target for PET diagnosis (patient PSMA phenotyping) and for targeted radionuclide therapy (2). In diagnostic PSMA PET, the easier availability of the ${ }^{68} \mathrm{Ga}$ tracer makes ${ }^{68} \mathrm{Ga}$-labeled ligands more commonly used in the clinic than ${ }^{18} \mathrm{~F}$-labeled PSMA compounds (3). However, there has also been a recent increase in the number of preclinical and clinical studies using ${ }^{18} \mathrm{~F}$-labeled PSMA ligands, such as ${ }^{18} \mathrm{~F}-\mathrm{PSMA}-1007,{ }^{18} \mathrm{~F}-\mathrm{DCFBC}(N-[N-[(S)-1$, 3-dicarboxypropyl]carbamoyl]-4- ${ }^{18} \mathrm{~F}$-fluorobenzyl-L-cysteine), and ${ }^{18}$ F-DCFPyL (2-(3-\{1-carboxy-5-[(6- ${ }^{18} \mathrm{~F}$-fluoro-pyridine-3-carbonyl)amino]-pentyl $\}$-ureido)-pentanedioic acid). Giesel et al. reported the superiority of ${ }^{18}$ F-PSMA-1007 for the diagnosis of pelvic metastases, focusing on its minimal uptake in the urinary tract (4). They also showed a good correlation between the results of pretreatment PET evaluation and distribution of the therapeutic agent ${ }^{177} \mathrm{Lu}-\mathrm{PSMA}-617$ (5).

In the field of neurology, it is well known that the concentration of the target molecule in a PET tracer solution affects its uptake. Because of competitive binding to the target, the presence of higher concentrations of the nonradiolabeled ligand can lower the uptake of the radiolabeled ligand. (6). However, this competitive effect can also be applied for dose optimization in drug development, using receptor occupancy tests in microdose PET examinations. In the field of oncology, de Jong et al. reported the effect of the amount of injected peptide on tissue distribution of ${ }^{111}$ In-labeled [DOTA ${ }^{0}$, $\mathrm{Tyr}^{3}$ ]octreotide, with uptake showing different bell-shaped functions depending on the organ and tumor (7). In addition, Konijnenberg et al. revealed that uptake of the cholecystokinin 2 compound PP-F11 in tumor showed a steep saturation effect with increasing amounts of peptide (8). In the case of PSMA radioligands, there have been a few published studies on how a variation in the 
TABLE 1

Profile of 3 Different MA Groups

\begin{tabular}{lcccc}
\hline \multicolumn{1}{c}{ Parameter } & High MA & Medium MA & Low MA & $P^{*}$ \\
\hline MA $(\mathrm{GBq} / \mu \mathrm{mol})$ & $1,013 \pm 146$ & $100.7 \pm 23.1$ & $10.8 \pm 2.84$ & $<0.05$ \\
\hline PSMA dose $(\mathrm{pmol})$ & $16.9 \pm 3.0$ & $179.9 \pm 28.5$ & $1,417 \pm 166$ & $<0.05$ \\
Injected activity $(\mathrm{MBq})$ & $16.8 \pm 2.0$ & $17.7 \pm 2.7$ & $15.0 \pm 2.8$ & 0.37 \\
Body weight $(\mathrm{g})$ & $18.4 \pm 1.9$ & $17.2 \pm 1.9$ & $18.5 \pm 2.3$ & 0.99 \\
Tumor volume $\left(\mathrm{mm}^{3}\right)$ & $114.5 \pm 93.3(82.0)$ & $73.0 \pm 35.7(77.2)$ & $61.3 \pm 43.3(62.4)$ & 0.44
\end{tabular}

${ }^{*}$ By independent $t$ test with Bonferroni correction.

Data are mean $\pm S D$, with median in parentheses.

peptide concentration or molar activity (MA) of the injected solution can affect uptake of the SPECT or PET radiotracer. Chatalic et al., in a preclinical model using SPECT, showed that coinjection of a PSMA inhibitor decreased the absorbed dose to the kidney and increased the tumor-to-kidney absorbed dose ratio during ${ }^{177}$ Lu-PSMA therapy (9). Also, Wurzer et al. recently reported that a coinjection of nonradiolabeled PSMA compound affects the uptake of ${ }^{68} \mathrm{Ga}$-labeled PSMA tracers in different organs and tissues to a highly varying degree (10). Different peptide concentrations of nonradiolabeled PSMA between diagnostic PET and radionuclide therapy might result in different degrees of radioligand accumulation in the target tissues and normal organs. In this study on mouse xenograft models, we evaluated changes in whole-body distribution using ${ }^{18}$ F-PSMA-1007 solutions with different peptide concentrations to optimize the MA level for subsequent targeted radionuclide therapy.

\section{MATERIALS AND METHODS}

\section{Synthesis and Preparation of 18F-PSMA-1007 Solutions}

${ }^{18}$ F-PSMA-1007 was synthesized by a 1-step method optimized using a MPS-200 cassette-type synthesizer (Sumitomo Heavy Industries), based on a previous report (11). In short, ${ }^{18} \mathrm{~F}-\mathrm{F}^{-}$produced by a Cypris-HM-18 cyclotron (Sumitomo Heavy Industries) was trapped on a quaternary methyl amine cartridge (carbonate form) and eluted into a reaction vessel with $0.075 \mathrm{M}$ tetrabutylammonium $\mathrm{HCO}_{3}$ solution $(600 \mu \mathrm{L})$. This solution was azeotropically dried with acetonitrile $(1 \mathrm{~mL})$. After the reaction vessel had been cooled, a dimethylsulfoxide solution of the PSMA-1007 precursor, 5-((S)-4-carboxy-1-((S)-4-carboxy1-(4- $((S)-1-((S)-5$-carboxy-5-(3- $((S)-1,3$-dicarboxypropyl)ureido) pentylamino)-3-(naphthalen-2-yl)-1-oxopropan-2-ylcarbamoyl)benzylamino)1-oxobutan-2-ylamino)-1-oxobutan-2-ylcarbamoyl)- $N, N, N$-trimethylpyridin2-aminium 2,2,2-acetate $(2 \mathrm{mg} / 2 \mathrm{~mL})$, was fed into the reaction vessel and fluorination was performed at $95^{\circ} \mathrm{C}$ for $10 \mathrm{~min}$. The reaction mixture was diluted with $5 \%$ ethanol solution $(10 \mathrm{~mL})$ and passed through PS- $\mathrm{H}^{+}$and $\mathrm{C} 18_{\text {ec }}$ cartridges. Then, these cartridges were washed with $5 \%$ ethanol solution $(23 \mathrm{~mL})$ and $30 \%$ ethanol solution $(3 \mathrm{~mL})$ for removal of any chemical or radiochemical impurities. ${ }^{18}$ F-PSMA-1007 trapped on the cartridge was finally eluted with $30 \%$ ethanol solution $(4 \mathrm{~mL})$ into the product vial containing $0.9 \% \mathrm{NaCl}$ solution $(11 \mathrm{~mL})$ plus $100 \mathrm{mg}$ of sodium ascorbate.

The radiochemical purity of ${ }^{18}$ F-PSMA-1007 was $96.9 \%-97.6 \%$, and the MA was $1,260-1,476 \mathrm{GBq} / \mu \mathrm{mol}$ at the end of the synthesis as analyzed with radio-high-performance liquid chromatography, which was performed on a Chromolith Performance RP-18e $(100 \times 4.6 \mathrm{~mm}$; Merck) using a Shimazu high-performance liquid chromatography system. Solvent A was $\mathrm{CH}_{3} \mathrm{CN}$, solvent $\mathrm{B}$ was $0.1 \%$ trifluoroacetic acid, and the gradient was as follows: A, $5 \%$ to $15 \%$ from 0 to $1.5 \mathrm{~min}$; A, $15 \%$ to $35 \%$ from 1.5 to $10.5 \mathrm{~min}$; A, $35 \%$ to $95 \%$ from 10.5 to $13 \mathrm{~min}$; and $\mathrm{A}, 95 \%$ to $5 \%$ from 13 to $19 \mathrm{~min}$ ); and $\mathrm{A}+\mathrm{B}=100 \%$, throughout). The flow rate was $3 \mathrm{~mL} / \mathrm{min}$, and the ultraviolet wavelength was $254 \mathrm{~nm}$.

The ${ }^{18}$ F-PSMA-1007 solution without dilution was used for injection in the high-MA group. For the medium-MA and low-MA groups, nonradiolabeled PSMA-1007 standard (ABX) was added to the ${ }^{18}$ F-PSMA- 1007 solutions $(1.5 \mu \mathrm{g}$ and $11 \mu \mathrm{g}$ of PSMA-1007 for $1 \mathrm{~mL}$ of each ${ }^{18} \mathrm{~F}$-PSMA-1007 solution). The final MA levels of the ${ }^{18}$ F-PSMA1007 solutions for the PET/CT study were $1,013 \pm 146 \mathrm{GBq} / \mu \mathrm{mol}$ (high MA), $100.7 \pm$ $23.1 \mathrm{GBq} / \mu \mathrm{mol}$ (medium MA), and $10.80 \pm$ $2.84 \mathrm{GBq} / \mu \mathrm{mol}$ (low MA), equivalent to doses of $16.9 \pm 3.0$ pmol, $179.9 \pm 28.5$ pmol, and $1,417 \pm 166$ pmol, respectively. For biodistribution experiments, the final MA levels were $863.6 \pm 64.3 \mathrm{GBq} / \mu \mathrm{mol}$ (high MA), $164.44 \pm$ $4.63 \mathrm{GBq} / \mu \mathrm{mol}$ (medium MA), and $23.69 \pm$ $0.75 \mathrm{GBq} / \mu \mathrm{mol}$ (low MA), equivalent to doses
FIGURE 1. (A-E) Immunohistochemical staining (high magnification) of tumor in high-MA group $(A)$, medium-MA group (B), low-MA group (C), kidney (D), and salivary gland (E), using anti-PSMA antibody. ( $F$ and $G$ ) Western blot analysis for evaluation of PSMA expression in high-, medium-, and low-MA groups (F) and in tumor, kidney, and salivary gland (G). M indicates molecular weight markers. Arrows indicate PSMA expression.
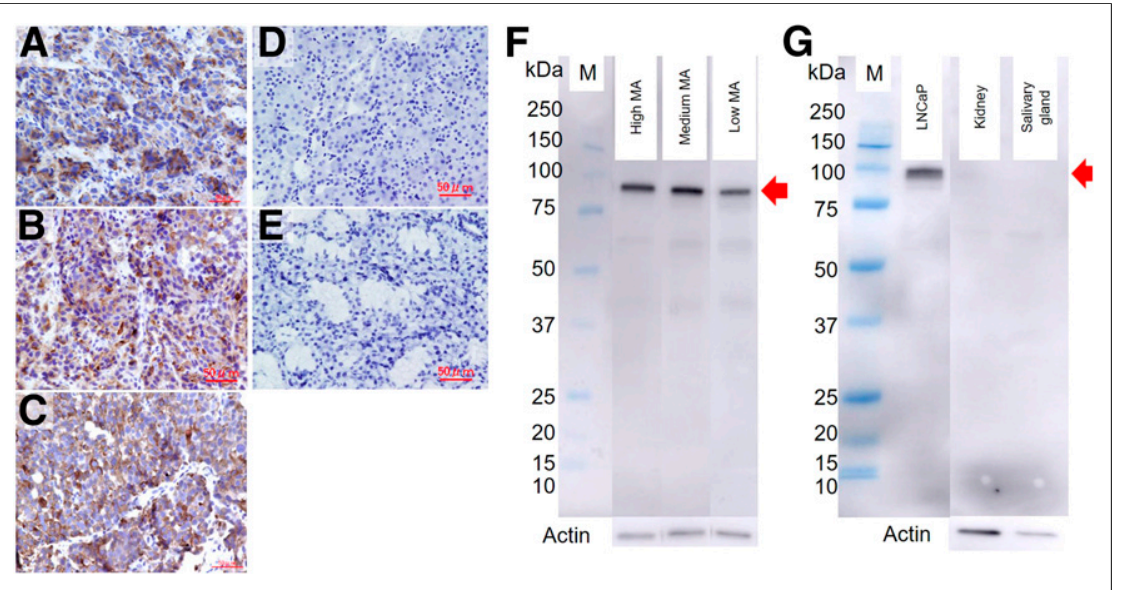


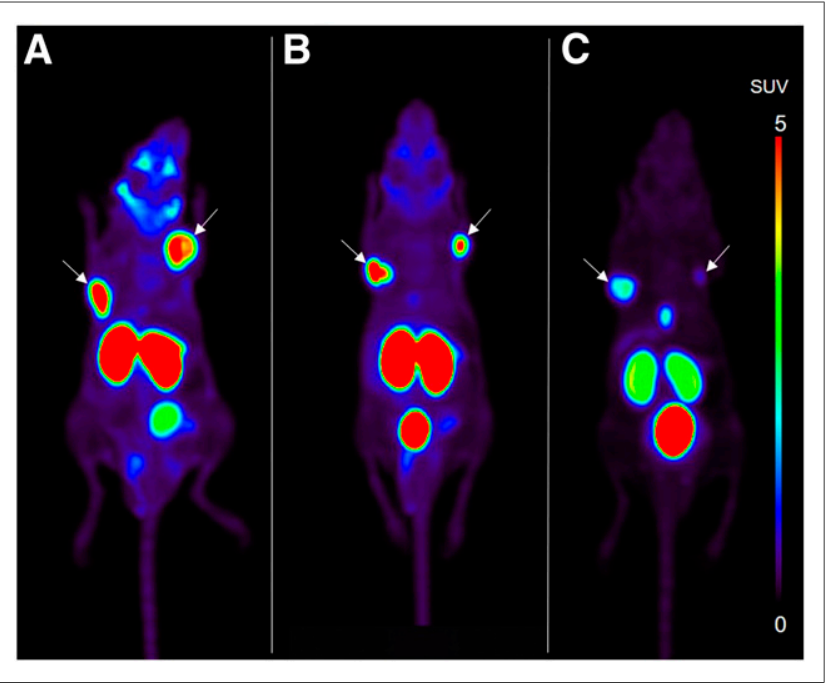

FIGURE 2. Maximum-intensity projections of ${ }^{18} \mathrm{~F}-\mathrm{PSMA}-1007$ PET in xenografts of mice of high-MA (A), medium-MA (B), and low-MA (C) groups. Arrows indicate tumors. Tumor uptake was lower in low-MA group than in medium- and high-MA groups.

of $5.11 \pm 0.22 \mathrm{pmol}, 25.65 \pm 0.49 \mathrm{pmol}$, and $174.8 \pm 19.4 \mathrm{pmol}$, respectively.

\section{Animal Preparation}

All animal experiments were performed under the guidelines of the Institute of Experimental Animal Sciences. The protocol was approved by the Animal Care and Use Committee of the Osaka University Graduate School of Medicine (approval 25-097-015). Male NOD/ SCID mice (4 wk old) were purchased from Charles River Japan, Inc. LNCaP, a prostate cancer cell line derived from a lymph node metastasis in a human, was obtained from Riken BRC. LNCaP cells were cultured in RPMI-1640 medium (Wako Pure Chemical Industries) containing $10 \%$ fetal bovine serum (Sigma-Aldrich) at $37^{\circ} \mathrm{C}$ in a humidified incubator containing $5 \% \mathrm{CO}_{2}$. To create the tumor xenograft models, $\mathrm{LNCaP}$ cells were collected in a 1:1 mixture $(\mathrm{v} / \mathrm{v})$ of medium and Matrigel (Corning) and subcutaneously implanted into either the left or right shoulder of the mice $\left(\sim 5 \times 10^{6}\right.$ cells were inoculated at each site). The tumor xenograft models ( $n=18$; body weight, $18.0 \pm$ $2.1 \mathrm{~g}$ ) were evaluated by small-animal PET/CT $40 \mathrm{~d}$ after implantation (tumor volume, $82.9 \pm 64.7 \mathrm{~mm}^{3}$ ). Male ICR mice ( $10 \mathrm{wk}$ old; $n=9$; body weight, $39.6 \pm 2.1 \mathrm{~g}$ ) were purchased from Japan SLC, Inc., and used as a non-tumor-bearing cohort for biodistribution experiments.

\section{PET/CT}

Data were acquired with a small-animal PET/CT system (Inveon) (12). The mice were anesthetized with an inhalational mixture of $2 \%$ isoflurane plus $100 \%$ oxygen, and ${ }^{18}$ F-PSMA-1007 (16.5 $\left.\pm 2.7 \mathrm{MBq}\right)$ was administered through the tail vein. One hour after the injection, and after CT, static PET scanning was performed for 10 min (13). All PET data were reconstructed by 3 -dimensional ordered-subset expectation maximization (16 subsets, 2 iterations), with correction for attenuation and scatter.

\section{Immunohistochemical Staining and Western Blot Analysis}

The tumor-bearing mice were euthanized and the tumors resected. Half the resected specimens were subjected to immunohistochemical staining using monoclonal mouse anti-human PSMA clone 3E6 (M3620; Dako) as the primary antibody and EnVision+ system (horseradish peroxidase) labeled polymer antimouse (K4001; Dako) as the secondary antibody, in accordance with the manufacturer's instructions (14). The other half of the tumor specimens was subjected to Western blot analysis, followed by the bicinchoninic acid assay, using anti-human PSMA, clone 3E6, monoclonal mouse antibody (M3620; Dako) as the primary antibody and rabbit polyclonal antibody to mouse IgG (horseradish peroxidase) (ab97046; Abcam) as the secondary antibody, in accordance with the manufacturer's instructions (15). Kidney and salivary gland obtained from male NOD/SCID mice ( $6 \mathrm{wk}$ ) were also subjected to immunohistochemical staining and Western blot analysis.

\section{Biodistribution Experiment}

ICR mice were divided into the high-MA $(n=3)$, medium-MA $(n=3)$, and low-MA $(n=3)$ groups. One hour after injection of solution prepared as described above, the mice were euthanized. Organs were collected, and the radioactivities of the samples were measured using a $\gamma$-counter (BeWell; Molecular Imaging Laboratory). The results were described as percentage injected dose.

\section{Data Analysis}

Regions of interest were placed on tumor, salivary gland, liver, gallbladder, kidney, urinary bladder, and muscle with reference to the fused CT images, using AMIDE software (version 1.0.4). Regional uptake of radioactivity was decay-corrected to the injection time and expressed as $\mathrm{SUV}_{\text {max }}$ and $\mathrm{SUV}_{\text {mean }}$, corrected for injected dose (MBq) and body weight $(\mathrm{g})$. SUV $\mathrm{SUan}_{\text {men }}$ for tumor and salivary gland was plotted against MA level of the injected radiotracer solution using the Michaelis-Menten model. The Michaelis-Menten constant $\left(\mathrm{K}_{\mathrm{M}}\right)$ and maximum rate $\left(\mathrm{V}_{\max }\right)$ were calculated using JMP software (version 14.0.0; SAS Institute Inc.). In addition, Scatchard analyses were

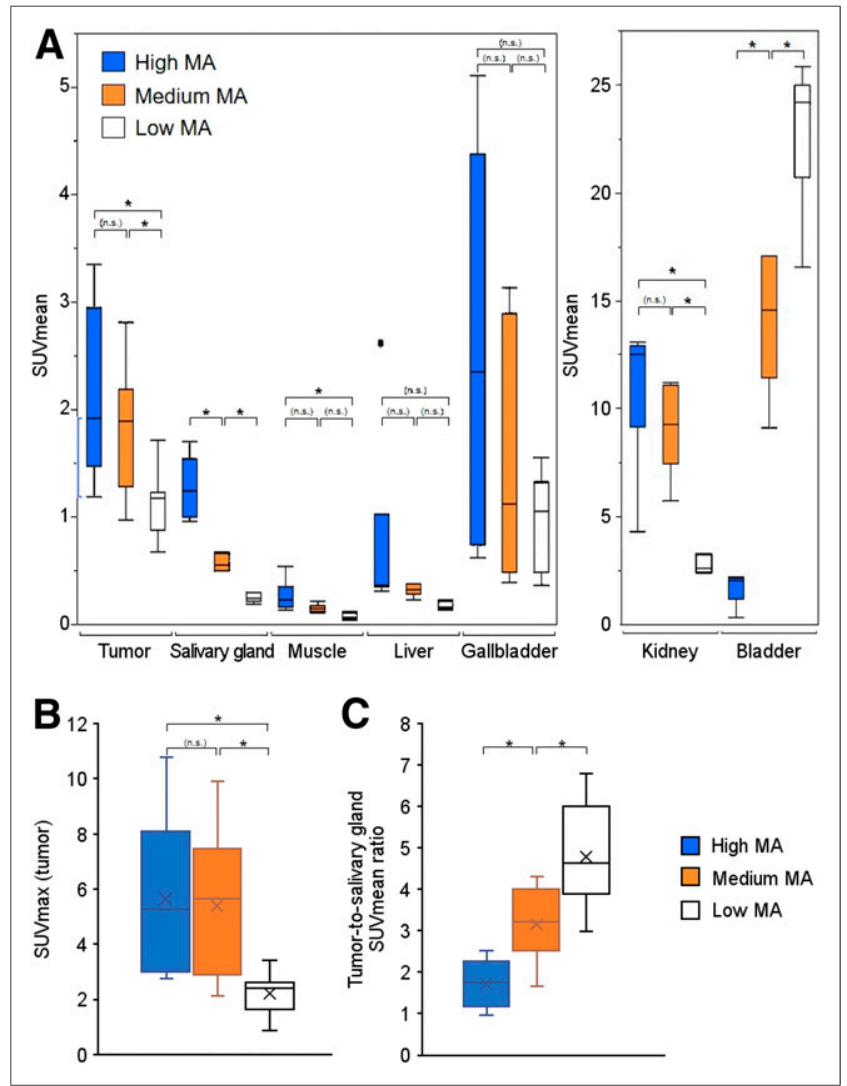

FIGURE 3. (A and B) $S U V_{\text {mean }}$ of major organs $(A)$ and $S U V_{\text {max }}$ of tumor (B) on ${ }^{18} \mathrm{~F}$-PSMA-1007 PET at 60 min after injection. (C) Ratios of SUV $\mathrm{V}_{\text {mean }}$ between tumor and salivary gland. ${ }^{*} P<0.05$. n.s. $=$ not significant. 
TABLE 2

Biodistribution of ${ }^{18} \mathrm{~F}-\mathrm{PSMA}-1007$ Solutions with Different Peptide Concentrations 60 Minutes After Administration in Non-Tumor-Bearing Mice

\begin{tabular}{lccc}
\hline \multicolumn{1}{c}{ Site } & High MA & Medium MA & Low MA \\
\hline Heart & $0.21 \pm 0.06$ & $0.17 \pm 0.02$ & $0.16 \pm 0.01$ \\
\hline Lung & $0.66 \pm 0.17$ & $0.56 \pm 0.13$ & $0.41 \pm 0.13$ \\
\hline Spleen & $0.95 \pm 0.21$ & $0.58 \pm 0.22$ & $0.45 \pm 0.22$ \\
\hline Liver & $0.82 \pm 0.09$ & $0.66 \pm 0.09$ & $0.99 \pm 0.24$ \\
\hline Kidney & $41.63 \pm 2.23$ & $41.90 \pm 1.25$ & $32.21 \pm 10.29$ \\
\hline Muscle & $0.10 \pm 0.03$ & $0.10 \pm 0.02$ & $0.10 \pm 0.01$ \\
\hline Brain & $0.01 \pm 0.00$ & $0.01 \pm 0.00$ & $0.01 \pm 0.00$ \\
\hline Salivary gland & $0.55 \pm 0.12$ & $0.35 \pm 0.04^{*}$ & $0.33 \pm 0.03^{*}$ \\
\hline Stomach & $0.21 \pm 0.03$ & $0.18 \pm 0.05$ & $0.22 \pm 0.03$ \\
\hline Pancreas & $0.22 \pm 0.06$ & $0.28 \pm 0.16$ & $0.24 \pm 0.03$ \\
\hline Testis & $0.26 \pm 0.05$ & $0.16 \pm 0.03^{*}$ & $0.17 \pm 0.01^{*}$ \\
\hline Small intestine & $2.10 \pm 0.29$ & $2.03 \pm 0.35$ & $1.92 \pm 0.31$ \\
\hline Large intestine & $0.44 \pm 0.08$ & $0.48 \pm 0.14$ & $0.50 \pm 0.17$ \\
\hline
\end{tabular}

${ }^{*} P<0.05$ compared with high-MA group by Tukey honestlysignificant-difference test.

Data are percentage injected dose.

performed to obtain maximal binding capacity $\left(\mathrm{B}_{\max }\right)$ and dissociation constant $\left(\mathrm{K}_{\mathrm{d}}\right)$ for tumor and salivary gland.

\section{Statistical Analysis}

JMP software and SPSS (version 25) were used for the statistical analyses, and the 3 groups were compared using the independent $t$ test with Bonferroni correction and the Tukey honestly-significant-difference test. A $P$ value of less than 0.05 was considered to indicate a significant difference.

\section{RESULTS}

The profiles of the 3 different MA groups are shown in Table 1. There were no significant differences in tumor size among
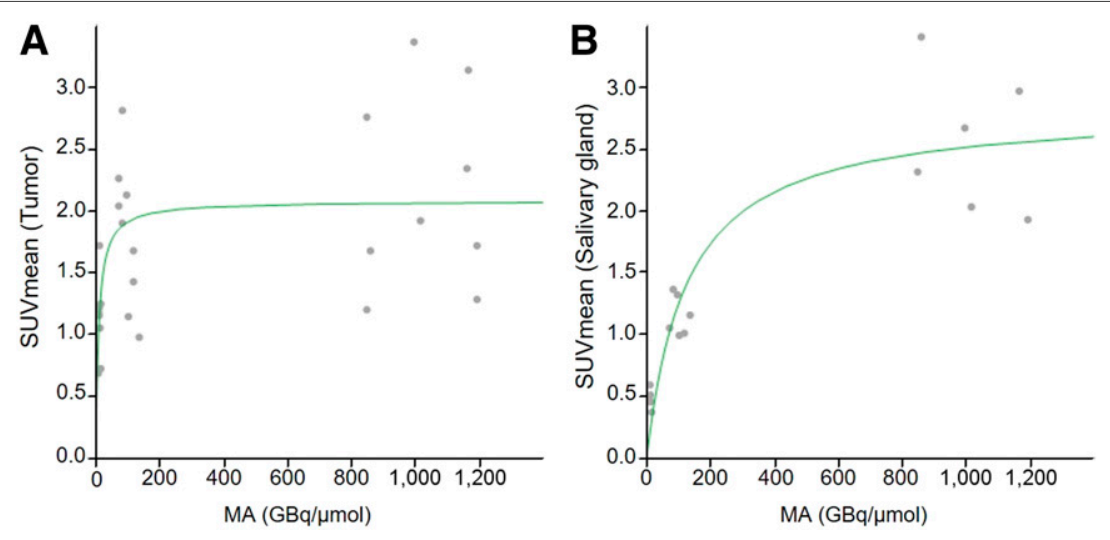

FIGURE 4. Michaelis-Menten curves showing relationship between MA level of injected solution and SUV in tumor (A) and salivary gland (B). $K_{M}$ and $V_{\max }$ were $8.78 \mathrm{GBq} / \mu \mathrm{mol}$ and $2.07 / \mathrm{h}$, respectively, in tumor and $126 \mathrm{GBq} / \mu \mathrm{mol}$ and $1.41 / \mathrm{h}$, respectively, in salivary gland. the 3 groups, although the larger trend was observed in the high-MA group because of the variability in size. The results of immunohistochemical staining for PSMA are shown in Figures 1A-1C. PSMA overexpression in the plasma membrane of the tumor cells was confirmed in tumor for all 3 groups. Western blot analysis also showed significant PSMA overexpression in tumor, with no significant differences among the groups (Fig. 1F). In contrast, salivary gland and kidney showed relatively low expression levels of PSMA, compared with tumor (Figs. 1D, 1E, and 1G).

${ }^{18}$ F-PSMA-1007 PET images are shown in Figure 2. In the high-MA group, uptake was high in tumor and kidney and moderate in salivary gland. In the medium-MA group, high uptake was preserved in tumor and kidney, but salivary gland uptake was lower than in the high-MA group. In the low-MA group, uptake in tumor, kidney, and salivary gland was lower than in the medium- and high-MA groups, but urinary excretion was higher. The volume-of-interest analysis showed significantly lower tumor uptake in the low-MA group $\left(\mathrm{SUV}_{\max }, 2.2 \pm 0.8\right)$ than in the high-MA group $\left(\mathrm{SUV}_{\max }, 5.7 \pm 2.9\right)$ and the medium-MA group $\left(\mathrm{SUV}_{\max }, 5.4 \pm 2.6\right)(P<0.05$ and $P<0.05$, respectively) (Figs. 3A and 3B). With regard to physiologic uptake in normal organs, salivary gland showed a more sensitive and dosedependent decrease in uptake as the MA level of the injected radiotracer solution was decreased. The tumor-to-salivary gland $\mathrm{SUV}_{\text {mean }}$ ratio increased with decreasing MA level of the injected radiotracer solution $(1.73 \pm 0.55$ in the high-MA group, $3.16 \pm 0.86$ in the medium-MA group, and $4.78 \pm 1.29$ in the low-MA group) (Fig. 3C). Urinary excretion of the radiotracer was significantly higher, and kidney and muscle uptake significantly lower, in the low-MA group than in the high- and medium-MA groups. On the other hand, no significant differences among the groups were observed for liver and gallbladder, although a trend toward decreased uptake in these organs was observed with a decrease in the MA level of the injected radiotracer solution.

The ${ }^{18}$ F-PSMA-1007 biodistribution in normal mice is shown in Table 2. Compared with the high-MA group, the medium- and lowMA groups showed a significant decrease in salivary gland and testis uptake.

Figure 4 shows the Michaelis-Menten curves for uptake in tumor and salivary gland. $\mathrm{K}_{\mathrm{M}}$ and $\mathrm{V}_{\max }$ were significantly lower for tumor $(8.78 \mathrm{GBq} / \mu \mathrm{mol}$ and $2.07 / \mathrm{h}$, respectively) than for salivary gland (126 $\mathrm{GBq} / \mu \mathrm{mol}$ and $1.41 / \mathrm{h}$, respectively). The results of the Scatchard plot analyses are shown in Figure 5 for tumor and salivary gland. $B_{\max }$ and $K_{d}$ were not estimated for salivary gland.

\section{DISCUSSION}

This study used PET and tissue sampling to evaluate the influence that different peptide concentrations in ${ }^{18} \mathrm{~F}$-PSMA-1007 injection solution have on tissue uptake. Uptake in major organs and tumor was significantly lower, and urinary excretion higher, in the low-MA group than in the medium- and high-MA groups.

Wurzer et al. recently reported that the MA levels of ${ }^{68} \mathrm{Ga}$-labeled PSMA inhibitor 


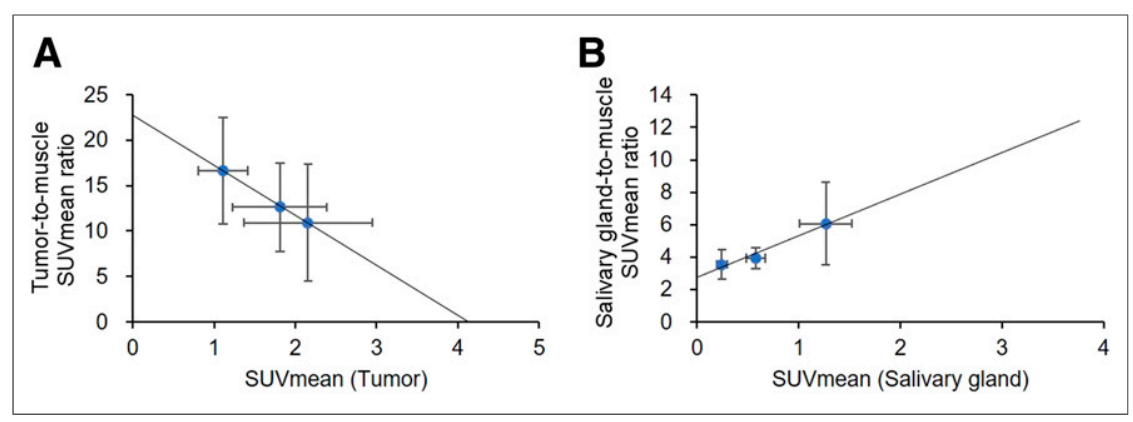

FIGURE 5. Scatchard plot analyses in tumor $(A)$ and salivary gland $(B)$. $B_{\max }$ and $K_{d}$ were 4.08 and 0.18 , respectively, in tumor. However, analysis failed to obtain $B_{\max }$ in salivary gland.

conjugates had a pronounced influence on the results of PSMA PET imaging $(9,10)$. In their study, addition of unlabeled compounds to the injection solution, which changed the MA levels from 1,200 to $8 \mathrm{MBq} / \mathrm{nmol}$, resulted in differential accumulation between tumor and normal organs such as kidney and salivary gland. They concluded that there is a need for careful evaluation of the influence of the nonradiolabeled mass or MA on the tumorto-organ uptake ratios of PSMA-targeted radiopharmaceuticals. Our findings are in line with these data, in that we found salivary gland uptake to be significantly decreased, but tumor uptake preserved, in the medium-MA group compared with the highMA group.

The $\mathrm{K}_{\mathrm{M}}$ for tumor in our study was much lower than that for salivary gland, suggesting that the binding affinity of ${ }^{18}$ F-PSMA1007 was higher in tumor than in salivary gland. As shown in Figure 1, the expression levels of PSMA in salivary gland were much lower than those in tumor. Previous studies reported that in contrast to the very low expression level of PSMA, kidney and salivary gland showed high physiologic uptake on PSMA PET $(1,4)$. In addition, Schottelius et al. recently reported that a very low expression level of PSMA was observed in kidney, compared with $\mathrm{LNCaP}$ tumor, by immunohistochemistry on mice (16). They speculated that non-PSMA-mediated uptake mechanisms, such as megalin/cubilin-mediated tubular reabsorption, were involved in the kidney uptake.

Xerostomia, caused by salivary gland uptake of the radioligand, is a major problem in targeted $\alpha$-therapy using ${ }^{225}$ Ac-PSMA-617; it significantly impairs the quality of life and sometimes even requires discontinuation of PSMA-targeted treatment $(17,18)$. The present study revealed that adjusting the MA level of the injected radiotracer can reduce salivary gland uptake while maintaining tumor uptake. Such an adjustment may become one practical option for preventing xerostomia during PSMA-targeted radionuclide therapy. ${ }^{18}$ F-PSMA-1007 PET studies with different MA levels in the solution should be performed before ${ }^{225}$ Ac-PSMA therapy to optimize the uptake ratio between tumor and salivary gland.

Renal dysfunction is a major problem in ${ }^{177} \mathrm{Lu}$-DOTATATE treatment because of the high kidney accumulation (19). In ${ }^{177} \mathrm{Lu}-\mathrm{PSMA}-617$ and ${ }^{225}$ Ac-PSMA-617 therapy, renal dysfunction has not been a major problem so far despite this high accumulation $(20,21)$. The present study revealed a high kidney accumulation of ${ }^{18}$ F-PSMA-1007, in parallel to the tumor accumulation. It would be difficult to adjust the MA level to decrease kidney accumulation while maintaining tumor accumulation. Therefore, in the clinical setting, renal dysfunction should be carefully monitored, particular before PSMAtargeted radionuclide therapy.

\section{CONCLUSION}

This study revealed that a decrease in the MA level of the injected ${ }^{18}$ F-PSMA-1007 solution resulted in decreased uptake in tumor and normal organs as seen on PET imaging and tissue sampling. Salivary gland was more sensitive than tumor to the decrease in MA level. This finding suggests the possibility of obtaining a therapeutic effect in tumor while minimizing adverse effects on salivary gland by setting an appropriate MA level in PSMA-targeted therapy. The optimal tracer dose might vary from patient to patient, depending on the tumor load and physiologic accumulation.

\section{DISCLOSURE}

Klaus Kopka and Frederik Giesel are listed as inventors on a PSMA-1007-relevant patent. This study was partly supported by collaborative research funds from Nihon Medi-Physics Co., Ltd., Japan. No other potential conflict of interest relevant to this article was reported.

\section{ACKNOWLEDGMENTS}

We thank the PET Drug Synthesis Department at Osaka University Hospital for preparing the tracers, the Medical Imaging Center for Translational Research for providing excellent technical assistance, the Department of Nuclear Medicine and Tracer Kinetics for supporting the experiments, and Nihon Medi-Physics Co., Ltd., for supporting the tumor sample analysis.

\section{KEY POINTS}

QUESTION: Does ${ }^{18} \mathrm{~F}-\mathrm{PSMA}-1007$ uptake in tumor and normal organs depend on its molar activity (MA) or peptide concentration of the injected solution?

PERTINENT FINDINGS: The whole-body distribution of ${ }^{18} \mathrm{~F}-$ PSMA-1007 on mouse xenograft models was evaluated when solutions with different peptide concentrations were used. A decrease in the MA level resulted in decreased uptake in tumor and, to a greater degree, in salivary gland.

IMPLICATIONS FOR PATIENT CARE: There is a possibility of obtaining a therapeutic effect in tumor while minimizing adverse effects in salivary gland by setting an appropriate MA level in PSMA-targeted therapy.

\section{REFERENCES}

1. Israeli RS, Powell CT, Corr JG, Fair WR, Heston WD. Expression of the prostate-specific membrane antigen. Cancer Res. 1994;54:1807-1811.

2. Herrmann K, Bluemel C, Weineisen $\mathrm{M}$, et al. Biodistribution and radiation dosimetry for a probe targeting prostate-specific membrane antigen for imaging and therapy. J Nucl Med. 2015;56:855-861.

3. Rahbar K, Weckesser M, Ahmadzadehfar H, Schafers M, Stegger L, Bogemann M. Advantage of ${ }^{18}$ F-PSMA-1007 over ${ }^{68}$ Ga-PSMA-11 PET imaging for differentiation 
of local recurrence vs. urinary tracer excretion. Eur J Nucl Med Mol Imaging. 2018;45:1076-1077.

4. Giesel FL, Hadaschik B, Cardinale J, et al. F-18 labelled PSMA-1007: biodistribution, radiation dosimetry and histopathological validation of tumor lesions in prostate cancer patients. Eur J Nucl Med Mol Imaging. 2017;44: $678-688$.

5. Giesel FL, Cardinale J, Schäfer M, et al. ${ }^{18}$ F-labelled PSMA-1007 shows similarity in structure, biodistribution and tumour uptake to the theragnostic compound PSMA-617. Eur J Nucl Med Mol Imaging. 2016;43:1929-1930.

6. Walker M, Ehrlichmann W, Stahlschmidt A, Pichler BJ, Fischer K. In vivo evaluation of ${ }^{11} \mathrm{C}$-DASB for quantitative SERT imaging in rats and mice. $\mathrm{J} \mathrm{Nucl}$ Med. 2016;57:115-121.

7. de Jong M, Breeman WA, Bernard BF, et al. Tumour uptake of the radiolabelled somatostatin analogue [DOTA0, TYR3] octreotide is dependent on the peptide amount. Eur J Nucl Med. 1999;26:693-698.

8. Konijnenberg MW, Breeman WA, de Blois E, et al. Therapeutic application of CCK2R-targeting PP-F11: influence of particle range, activity and peptide amount. EJNMMI Res. 2014;4:47.

9. Chatalic KL, Heskamp S, Konijnenberg M, et al. Towards personalized treatment of prostate cancer: PSMA I\&T, a promising prostate-specific membrane antigentargeted theranostic agent. Theranostics. 2016;6:849-861.

10. Wurzer A, Pollmann J, Schmidt A, Reich D, Wester HJ, Notni J. Molar activity of Ga-68 labeled PSMA inhibitor conjugates determines PET imaging results. Mol Pharm. 2018;15:4296-4302.

11. Cardinale J, Martin R, Remde Y, et al. Procedures for the GMP-compliant production and quality control of $\left[{ }^{18} \mathrm{~F}\right]$ PSMA-1007: a next generation radiofluorinated tracer for the detection of prostate cancer. Pharmaceuticals (Basel). 2017;10:E77.

12. Bao Q, Newport D, Chen M, Stout DB, Chatziioannou AF. Performance evaluation of the Inveon dedicated PET preclinical tomograph based on the NEMA NU-4 standards. J Nucl Med. 2009;50:401-408.
13. Cardinale J, Schafer M, Benesova M, et al. Preclinical evaluation of ${ }^{18}$ F-PSMA1007 , a new prostate-specific membrane antigen ligand for prostate cancer imaging. J Nucl Med. 2017;58:425-431.

14. Silver DA, Pellicer I, Fair WR, Heston WD, Cordon-Cardo C. Prostate-specific membrane antigen expression in normal and malignant human tissues. Clin Cancer Res. 1997;3:81-85.

15. Troyer JK, Beckett ML, Wright GL Jr. Detection and characterization of the prostate-specific membrane antigen (PSMA) in tissue extracts and body fluids. Int J Cancer. 1995;62:552-558.

16. Schottelius M, Wurzer A, Wissmiller K, et al. Synthesis and preclinical characterization of the PSMA-targeted hybrid tracer PSMA-I\&F for nuclear and fluorescence imaging of prostate cancer. $J$ Nucl Med. 2019;60:71-78.

17. Kratochwil C, Bruchertseifer F, Rathke H, et al. Targeted alpha-therapy of metastatic castration-resistant prostate cancer with ${ }^{225}$ Ac-PSMA-617: swimmer-plot analysis suggests efficacy regarding duration of tumor control. J Nucl Med. 2018;59: 795-802.

18. Kratochwil C, Bruchertseifer F, Giesel FL, et al. ${ }^{225}$ Ac-PSMA-617 for PSMAtargeted alpha-radiation therapy of metastatic castration-resistant prostate cancer. J Nucl Med. 2016;57:1941-1944.

19. Demirci E, Kabasakal L, Toklu T, et al. ${ }^{177}$ Lu-DOTATATE therapy in patients with neuroendocrine tumours including high-grade (WHO G3) neuroendocrine tumours: response to treatment and long-term survival update. Nucl Med Commun. 2018;39:789-796.

20. Kratochwil C, Bruchertseifer F, Rathke H, et al. Targeted alpha-therapy of metastatic castration-resistant prostate cancer with ${ }^{225}$ Ac-PSMA-617: dosimetry estimate and empiric dose finding. J Nucl Med. 2017;58:1624-1631.

21. Hofman MS, Violet J, Hicks RJ, et al. $\left[{ }^{177} \mathrm{Lu}\right]-P S M A-617$ radionuclide treatment in patients with metastatic castration-resistant prostate cancer (LuPSMA trial): a single-centre, single-arm, phase 2 study. Lancet Oncol. 2018;19:825833. 